\title{
Predictors of Mitral Regurgitation Severity Improvement in Patients With Severe Aortic Stenosis Undergoing Transcatheter Aortic Valve Implantation
}

\author{
Alla Lubovich ${ }^{\mathrm{a}, \mathrm{b}, \mathrm{c}}$, Fabio Kusniec ${ }^{\mathrm{a}, \mathrm{b}}$, Doron Sudarskya, ${ }^{\mathrm{a}}$, \\ Liza Grosman-Rimon ${ }^{\mathrm{a}, \mathrm{b}}$, Shemy Carasso ${ }^{\mathrm{a}, \mathrm{b}}$
}

\begin{abstract}
Background: Mitral regurgitation (MR) is frequently associated with severe aortic stenosis (AS). Significant MR is associated with less favorable prognosis after transcatheter aortic valve implantation (TAVI), including higher early and late mortality rate. The severity of MR is improved in about half of patients undergoing TAVI. However, the predictors of MR improvement after TAVI are unknown. We sought to investigate whether several demographic, clinical, echocardiographic and laboratory parameters and procedure characteristics are predictive of MR severity improvement after TAVI procedure.
\end{abstract}

Methods: A total of 309 consecutive patients with severe symptomatic AS underwent TAVI procedure in our center from July 1, 2015 till December 31, 2019. The 85 patients had concomitant significant (grade 2 or 3) MR. We performed logistic regression analysis of age, sex, atrial fibrillation, left ventricular ejection fraction, end diastolic diameter, end systolic diameter, left atrial diameter, left atrial area, MR etiology (functional vs. degenerative), CHA2DS2-VASc score, pre-procedure B-type natriuretic peptide (BNP) levels and type of TAVI bioprosthesis as possible predictors of post-TAVI improvement of severity of MR.

Results: The 35 patients have at least one grade reduction in the severity of MR in follow-up echo. None of the analyzed parameters were predicting of the MR severity improvement.

Conclusions: In this small single-center cohort study, we were unable to find any feasible demographic, clinical, echocardiographic or laboratory predictors of MR improvement after TAVI. There was no correlation between etiology of MR or type of TAVI bioprosthesis used and MR improvement.

Manuscript submitted September 27, 2020, accepted October 30, 2020

Published online December 11, 2020

${ }^{a}$ Cardiovascular Institute, B Padeh Medical Center, Poriya, Israel

${ }^{b}$ The Azrieli Faculty of Medicine in the Galilee, Bar-Ilan University, Safed, Israel

${ }^{\mathrm{c}}$ Corresponding Author: Alla Lubovich, Cardiovascular Institute, B Padeh Medical Center, Poriya, Israel. Email: dr.allal@gmail.com

doi: https://doi.org/10.14740/cr1174
Keywords: TAVI; Mitral regurgitation; Echocardiography

\section{Introduction}

Mitral regurgitation (MR) is frequently associated with severe aortic stenosis (AS). Previous studies have demonstrated that about one-third of patients undergoing transcatheter aortic valve implantation (TAVI) for the treatment of severe AS, have at least moderate MR (MR grade $\geq 2$ ) [1-4]. Significant MR is associated with less favorable prognosis after TAVI, including higher early and late mortality rate [4-8]. Several previous studies had conflicted results about incidence of MR improvement in patients with severe AS after TAVI procedure, with numbers ranging from $30 \%$ to $70 \%$ [4-8]. Moreover, the predictors of MR improvement after TAVI are largely unknown. As there is a worldwide acceptance of TAVI as an alternative to surgical aortic valve replacement not only in patients with high surgical risk but also in those with intermediate and low surgical risk, the ability to predict MR severity improvement before the procedure is becoming crucial. We sought to investigate whether several demographic, clinical, echocardiographic and laboratory parameters and procedure characteristics are predictive of MR severity improvement after TAVI procedure.

\section{Materials and Methods}

It was a single-center prospective cohort study based on our institutional TAVI registry. The study was approved by the Institutional Review Board and conducted in compliance with the ethical standards of the Helsinki Declaration.

\section{Patients}

Three hundred and nine $(\mathrm{n}=309)$ consecutive patients with severe symptomatic AS underwent TAVI procedure in our center from July 1, 2015 till December 31, 2019. Eighty-five patients $(\mathrm{n}=85)$ had concomitant significant (grade 2 or 3 ) MR on preprocedure echo and were included in the analyses. The MR 
Table 1. Baseline Clinical Characteristics

\begin{tabular}{ll}
\hline Characteristics & \\
\hline $\mathrm{N}$ & 85 \\
Age, mean (SD) & $80.9(0.72)$ \\
Male sex, \% & 47.1 \\
\hline Body mass index, mean (SD) & $27.72(0.65)$ \\
\hline Hypertension, \% & 91 \\
Hyperlipidemia, \% & 72 \\
\hline Diabetes mellitus, \% & 47 \\
\hline Current smoking, \% & 9 \\
\hline
\end{tabular}

SD: standard deviation.

severity was defined either by semiquantitative (the width of vena contracta) or quantitative (the effective regurgitant orifice) analysis. Baseline clinical characteristics of the patients are described in Table 1.

\section{Pre-procedure echo characteristics}

We performed analysis of several echo parameters including left ventricular systolic function, left ventricular systolic and diastolic diameter, left atrial diameter and area, and left ventricular diastolic function (Table 2).

\section{Laboratory parameters}

We performed analysis of pre-procedure pro-B-type natriuretic peptide (BNP). The descriptive statistics is shown in Table 2.

\section{Type of TAVI bioprosthesis used}

We used Evolute, Sapien and Accurate Neo valves. We analyzed whether type of TAVI bioprosthesis is correlated with MR improvement. The descriptive statistics is shown in Table 2.

\section{Atrial fibrillation and CHA2DS2-VASc score}

We analyzed whether atrial fibrillation and CHA2DS2-VASc score are correlated with MR improvement/non-improvement. The descriptive statistics is shown in Table 2.

\section{Statistical analysis}

All data were analyzed with SPSS software, Version 18.0 (SPSS Inc., Chicago, IL, USA). Categorical variables were presented as frequency (percentage) and or median \pm interquartile range (IQR). Continuous variables were presented as mean \pm standard deviation (SD). A Chi-square test was performed to assess the differences of 30-day and 1-year mortality in patients with improved and not improved MR. To further evaluate the independent predictors of MR, a univariable logistic regression analysis was performed. Using the backwards logistic regression model, we utilized a backwards variable elimination process. We began by assessing the statistical significance of the univariate association between each covariate and the outcome. All covariates whose univariate statistical significance was $<0.1$ were forced into a multivariable model. Backwards variable elimination was then used to develop a parsimonious regression model. Those variables whose adjusted statistical significance was $<0.175$ were retained in the final model. Odds ratio (OR) with a 95\% confidence interval

Table 2. Descriptive Statistics of Analyzed Echo, Laboratory, Clinical Parameters and Procedure Parameters

\begin{tabular}{ll}
\hline Parameters & \\
\hline BNP, mean \pm SD & $1,035 \pm 1,000$ \\
CHA2DS2-VASc & $5.1 \pm 1.3$ \\
Atrial fibrillation, $\%$ & 44.7 \\
LVEF, mean \pm SD & $53 \pm 13$ \\
LVEDD, mean \pm SD & $53.7 \pm 6.7$ \\
LVESD, mean \pm SD & $38.1 \pm 7.4$ \\
LA area, mean \pm SD & $25.5 \pm 5.5$ \\
LA diameter, mean \pm SD & $46.3 \pm 6.4$ \\
Moderate to severe diastolic dysfunction (grade 2, 3), \% & 44 \\
Functional MR, $\%$ & 64 \\
Evolute, $\%$ & 74 \\
Sapien, $\%$ & 18 \\
Accurate Neo, $\%$ & 8 \\
\hline
\end{tabular}

BNP: B-type natriuretic peptide; SD: standard deviation; LVEF: left ventricular ejection fraction; LVEDD: left ventricular end diastolic diameter; LVESD: left ventricular end systolic diameter; LA: left atrial; MR: mitral regurgitation. 
Table 3. Results of Logistic Regression Analysis of Possible Predictors of Post TAVI MR Severity Improvement

\begin{tabular}{lllll}
\hline Variables & B & P & OR & 95\% CI \\
\hline Age & 0.050 & 0.209 & 1.051 & $0.973-1.136$ \\
Sex (male) & 0.405 & 0.428 & 0.667 & $0.245-1.818$ \\
LVEF & 0.006 & 0.761 & 1.006 & $0.967-1.047$ \\
LVESD & -0.003 & 0.940 & 0.997 & $0.930-1.070$ \\
LVEDD & 0.007 & 0.877 & 1.007 & $0.924-1.097$ \\
LA area & -0.060 & 0.350 & 0.942 & $0.830-1.068$ \\
LA diameter & 0.003 & 0.941 & 1.003 & $0.927-1.086$ \\
Moderate to severe diastolic dysfunction & -0.036 & 0.779 & 0.92 & $0.881-1.077$ \\
Functional MR & -0.923 & 0.079 & 0.397 & $0.142-1.113$ \\
Atrial fibrillation & 0.431 & 0.398 & 1.538 & $0.566-4.181$ \\
CHA2DS2-VASc & -0.091 & 0.671 & 0.913 & $0.600-1.390$ \\
Pro-BNP & 0.000 & 0.200 & 1.000 & $0.999-1.000$ \\
Valve type & 0.37 & 0.346 & 1.003 & $0.997-1.009$ \\
\hline
\end{tabular}

TAVI: transcatheter aortic valve implantation; MR: mitral regurgitation; OR: odds ratio; Cl: confidence interval; LVEF: left ventricular ejection fraction; LVEDD: left ventricular end diastolic diameter; LVESD: left ventricular end systolic diameter; LA: left atrial; BNP: B-type natriuretic peptide.

(CI) and P values were derived from the Wald Chi-square test. Sensitivity and specificity were computed for BNP using the median as the cut-point. Statistical significance was considered to be two-sided $\mathrm{P}$ values of $<0.05$.

\section{Results}

At baseline, before the TAVI procedure, 70 patients have moderately severe (grade 2) MR and 15 patients have severe MR (grade 3). Thirty-five patients had at least one grade improvement in severity of MR in follow-up echo performed 3 months after the procedure. None of the analyzed echo, laboratory or clinical parameters were predictive of MR severity improvement after the TAVI procedure. There was no correlation between the etiology of MR (functional vs. degenerative) or type of TAVI valve used (Table 3 ). The 30-day and 1-year mortality of the total TAVI cohort $(\mathrm{n}=309)$ was $4.8 \%$ and $11.9 \%$, respectively. There was no difference in 30 -day $(0 \%$ vs. $1.6 \%, \mathrm{P}$ $=0.435)$ and 1 -year $(8.6 \%$ vs. $18.5 \%, P=0.247)$ mortality in patients with improved and not improved MR.

\section{Discussion}

In this small single-center study, we demonstrated that about $40 \%$ of patients with moderate to severe MR have at least one grade improvement in the severity of the MR 3 months after TAVI. This is compatible with data in previously published studies [4-8]. We were unable to find any meaningful predictor of MR severity improvement among very broad range of clinical, echocardiographic and laboratory parameters. There was no correlation between the type of TAVI valve implanted and the MR severity improvement. This finding is contradictory to a small echocardiographic study of Giordana et al [9], which demonstrated that reduction in MR was significant in patients treated with the Edwards Sapien device but not in patients treated with the CoreValve device.

\section{Limitations}

This is a small retrospective single-center study.

\section{Conclusions}

We were unable to find any meaningful predictor of MR severity improvement in patients with moderate to severe MR undergoing TAVI. However, there was no increased 1-year mortality in patients with not improved MR. Currently, the decision to proceed with TAVI in low or intermediate surgical risk patients with severe AS and concomitant significant MR should be made on individual basis after careful heart team discussion.

\section{Acknowledgments}

None to declare.

\section{Financial Disclosure}

None to declare.

\section{Conflict of Interest}

None to declare. 


\section{Informed Consent}

Not applicable.

\section{Authors Contributions}

Alla Lubovich: substantial contribution to the conception and design of the manuscript, drafting of the manuscript and final approval of the version to be published. Fabio Kusniec: substantial contribution to the conception of the manuscript, critical revising of the manuscript and final approval of the version to be published. Doron Sudarsky: substantial contribution to the conception of the manuscript, critical revising of the manuscript and final approval of the version to be published. Liza Grossman-Rimon: analysis and interpretation of the data for the manuscript, critical revising of the manuscript and final approval of the version to be published. Shemy Carasso: substantial contribution to the conception of the manuscript, critical revising of the manuscript and final approval of the version to be published.

\section{Data Availability}

The data supporting the findings of this study are available from the corresponding author on request.

\section{References}

1. Gurvitch R, Wood DA, Tay EL, Leipsic J, Ye J, Lichtenstein SV, Thompson CR, et al. Transcatheter aortic valve implantation: durability of clinical and hemodynamic outcomes beyond 3 years in a large patient cohort. Circulation. 2010;122(13):1319-1327.
2. Leon MB, Smith CR, Mack M, Miller DC, Moses JW, Svensson LG, Tuzcu EM, et al. Transcatheter aortic-valve implantation for aortic stenosis in patients who cannot undergo surgery. N Engl J Med. 2010;363(17):1597-1607.

3. Webb JG, Pasupati S, Humphries K, Thompson C, Altwegg L, Moss R, Sinhal A, et al. Percutaneous transarterial aortic valve replacement in selected high-risk patients with aortic stenosis. Circulation. 2007;116(7):755-763.

4. Muratori M, Fusini L, Tamborini G, Ghulam Ali S, Gripari P, Fabbiocchi F, Salvi L, et al. Mitral valve regurgitation in patients undergoing TAVI: Impact of severity and etiology on clinical outcome. Int J Cardiol. 2020;299:228234.

5. Sannino A, Losi MA, Schiattarella GG, Gargiulo G, Perrino C, Stabile E, Toscano E, et al. Meta-analysis of mortality outcomes and mitral regurgitation evolution in 4,839 patients having transcatheter aortic valve implantation for severe aortic stenosis. Am J Cardiol. 2014;114(6):875882.

6. Khawaja MZ, Williams R, Hung J, Arri S, Asrress KN, Bolter K, Wilson K, et al. Impact of preprocedural mitral regurgitation upon mortality after transcatheter aortic valve implantation (TAVI) for severe aortic stenosis. Heart. 2014;100(22):1799-1803.

7. Mavromatis K, Thourani VH, Stebbins A, Vemulapalli S, Devireddy C, Guyton RA, Matsouaka R, et al. Transcatheter aortic valve replacement in patients with aortic stenosis and mitral regurgitation. Ann Thorac Surg. 2017;104(6):1977-1985.

8. Chakravarty T, Van Belle E, Jilaihawi H, Noheria A, Testa L, Bedogni F, Ruck A, et al. Meta-analysis of the impact of mitral regurgitation on outcomes after transcatheter aortic valve implantation. Am J Cardiol. 2015;115(7):942-949.

9. Giordana F, Capriolo M, Frea S, Marra WG, Giorgi M, Bergamasco L, Omede PL, et al. Impact of TAVI on mitral regurgitation: a prospective echocardiographic study. Echocardiography. 2013;30(3):250-257. 